\title{
The SME gauge sector with minimum length
}

\author{
H. Belich ${ }^{\mathrm{a}}$, H. L. C. Louzada ${ }^{\mathrm{b}}$ \\ Departamento de Física e Química, Universidade Federal do Espírito Santo, Vitória, ES 29060-900, Brazil
}

Received: 30 April 2017 / Accepted: 1 December 2017 / Published online: 15 December 2017

(C) The Author(s) 2017. This article is an open access publication

\begin{abstract}
We study the gauge sector of the Standard Model Extension (SME) with the Lorentz covariant deformed Heisenberg algebra associated to the minimum length. In order to find and estimate corrections, we clarify whether the violation of Lorentz symmetry and the existence of a minimum length are independent phenomena or are, in some way, related. With this goal, we analyze the dispersion relations of this theory.
\end{abstract}

\section{Introduction}

The possibility of Lorentz symmetry violation (LV) induced by fluctuations which grow up when we investigate new theories that aim to reach the Planck scale physics has become nowadays an interesting research issue. LV has been investigated in two major proposed scenarios: spontaneous Lorentz symmetry violation (LSV) induced by a tensor background, and the breaking triggered by the generalization of the uncertainty principle - non-commutative geometry.

The proposal of LSV has appeared in a seminal article by Kostelecký and Samuel [1], giving rise to a line of investigation of possible physics beyond the Standard Model (SM). They have suggested, in a scenario of open string field theory, that LSV may occur through non-scalar fields (actually, through the vacuum condensation of fields that have a tensor nature) $[2,3]$. Therefore, a more fundamental theory could produce signals from more fundamental fields through LSV. This extension of the SM, which it is called the Standard Model Extension (SME), keeps gauge invariance, the conservation of energy and momentum and the covariance under observer rotations and boosts [4]. In this context, it is well known that the presence of terms which violate Lorentz symmetry selects at least one privileged direction in spacetime. In recent decades, studies of the Lorentz symmetry violation

\footnotetext{
a e-mail: belichjr@gmail.com

be-mail: haofisica@bol.com.br
}

have been pushed forward in several branches of physics [528].

The proposal of non-commutative geometry had already been mentioned by Heisenberg in 1930, in a letter to his student Rudolf Peierls, and it took 17 years until Snyder, in 1947 [29], put in practice this idea. In the same year, Yang made the proposal of a curved spacetime quantization (de Sitter universe) [30]. In 1980, Connes picked up this idea [31] and realizes that the non-commutative geometry would be a new scheme to extend the standard model $[32,33]$. The most recent proposal appears naturally in the context of string theory [34]. In this way, we have obtained an effective theory derived from string theory, which, in the low energy limit, reproduces some known physics.

In fact, our proposal to investigate LSV from the viewpoint of a minimum length is a natural way investigated in the literature with a long history. For example, there is a theory called doubly special relativity, with also introduces a minimum length as a new fundamental constant, in addition to the light speed $c$, to study LSV $[35,36]$. Such a viewpoint for a minimum length is also emphasized in recent studies: of an analysis of black hole entropy with minimum length [37], and a study of a new proposal for LV from basic considerations [38].

A possible way to explore the implementation of noncommutative theories is by the deformation of the Heisenberg algebra. In this paper, we study a modified Heisenberg algebra, by adding certain small corrections to the canonical commutation relations. It leads, as shown by Kempf et al. [39-43], to a minimum uncertainty in the position measurement, $\Delta x_{0}$, called the minimum length [44-46].

The hydrogen atom is one of the simplest quantum systems that allows for theoretical predictions of high accuracy, and a non-relativistic study is well established experimentally, offering a vast collection of highly precise measurements [47]. There are many references where the energy spectrum of the hydrogen atom in the presence of a minimum length 
is worked out [48-50], some of which have a divergence at the level $s(n=1)$ [49]

Recently, Quesne and Tkachuk have introduced a Lorentz covariant deformed algebra that describes a quantized $D+1$ dimensional spacetime [51,52]. It is given by the following generalized commutation relations:

$$
\begin{aligned}
& {\left[X^{\mu}, P^{\nu}\right]=-i \hbar\left[\left(1-\beta P_{\rho} P^{\rho}\right) g^{\mu \nu}\right.} \\
& \left.-\beta^{\prime} P^{\mu} P^{\nu}\right], \quad\left[P^{\mu}, P^{\nu}\right]=0, \\
& {\left[X^{\mu}, X^{\nu}\right]} \\
& \quad=i \hbar \frac{\left[\left(2 \beta-\beta^{\prime}\right)-\left(2 \beta+\beta^{\prime}\right) \beta P_{\rho} P^{\rho}\right]\left(P^{\mu} X^{\nu}-P^{\nu} X^{\mu}\right)}{\left(1-\beta P_{\rho} P^{\rho}\right)},
\end{aligned}
$$

where $\mu, v, \rho=0,1, \ldots, D, g_{\mu \nu}=g^{\mu \nu}=\operatorname{diag}(1,-1,-1$, $\ldots,-1), \beta$ and $\beta^{\prime}$ are deformation parameters, and we assume $\beta, \beta^{\prime}>0$. From the uncertainty relation, we conclude that the minimum length is

$$
\begin{aligned}
& \left(\Delta X^{i}\right)_{0}=\hbar \sqrt{\left(D \beta+\beta^{\prime}\right)\left[1-\beta\left\langle\left(P^{0}\right)^{2}\right\rangle\right]}, \\
& \forall i \in\{1, \ldots, D\} .
\end{aligned}
$$

An algebraic representation [53] satisfying (1) to first order in $\beta, \beta^{\prime}$ is given by

$$
\begin{aligned}
X^{\mu} & =x^{\mu}-\frac{\left(2 \beta-\beta^{\prime}\right)}{4}\left(x^{\mu} p_{\rho} p^{\rho}+p_{\rho} p^{\rho} x^{\mu}\right), \\
P^{\mu} & =\left(1-\frac{\beta^{\prime}}{2} p_{\rho} p^{\rho}\right) p^{\mu},
\end{aligned}
$$

where $x^{\mu}$ and $p^{\mu}=i \hbar \partial^{\mu}$ are respectively the position and momentum operators. Assuming $\beta^{\prime}=2 \beta$, the QuesneTkachuk algebra becomes

$$
\begin{aligned}
& {\left[X^{\mu}, P^{\nu}\right]=-i \hbar\left[\left(1-\beta P_{\rho} P^{\rho}\right) g^{\mu \nu}-2 \beta P^{\mu} P^{\nu}\right],} \\
& {\left[P^{\mu}, P^{\nu}\right]=0,} \\
& {\left[X^{\mu}, X^{\nu}\right]=0,}
\end{aligned}
$$

with the following representations that satisfy (4) to first order in $\beta$ :

$$
\begin{aligned}
& X^{\mu}=x^{\mu}, \\
& P^{\mu}=\left(1-\beta^{\prime} p_{\rho} p^{\rho}\right) p^{\mu} .
\end{aligned}
$$

In this paper, we investigate a scenario of anisotropy generated by a Lorentz symmetry breaking term which appears in the Standard Model Extension (SME) [54,55]. Also, we investigate the effects of a minimal length given by the deformation of the Heisenberg algebra and we study the influence of the anisotropy generated in both ways. The structure of this paper is as follows: in Sect. 2, we introduce the gauge sector of the SME. In Sects. 3 and 4, we shall study, respectively, the CPT-even and -odd gauge sectors of the SME in the presence of a minimum length. In Sect. 5, we present our concluding remarks.

\section{The gauge sector of the Standard Model Extension}

The Standard Model Extension (SME) is an effective field theory obtained from the Standard Model (the model that uniquely describes the interactions that govern elementary particles, namely electromagnetic, weak nuclear and strong nuclear interactions, but does not incorporate gravity) by the addition of terms that incorporate the violations of the Lorentz and CPT symmetry [56,57]. The added terms are constructed by the contraction of field operators of the Standard Model with tensor coupling constants that appear as a background in spacetime. These tensor coefficients (or coupling constants) have their origins in a more fundamental theory, where the LSV occurs, the coupling constants being used to build up the SME action; they appear as vacuum expectation values of the fundamental tensor fields. There are plenty of terms that can be constructed in this way, including non-renormalizable terms of arbitrarily high dimensions [23,58-60].

The Lagrangian density of the SME gauge sector [56,57] is given by

$$
\begin{aligned}
\mathcal{L} & =-\frac{1}{4 \mu_{0}} F_{\mu \nu} F^{\mu \nu}-\frac{\chi}{4 \mu_{0}} \epsilon_{\mu \nu \alpha \beta} V^{\mu} A^{\nu} F^{\alpha \beta} \\
& -\frac{1}{4 \mu_{0}}\left(K_{F}\right)_{\mu \nu \kappa \lambda} F^{\mu \nu} F^{\kappa \lambda}-A_{\mu} J^{\mu},
\end{aligned}
$$

with $F^{\mu v}=\partial^{\mu} A^{v}-\partial^{v} A^{\mu}$ the tensor of the electromagnetic field constructed from the gauge field $A^{\mu}=\left(A^{0}, \vec{A}\right)$.

The LV considered here is parametrized by the tensor field background $V^{\mu}$ and $\left(K_{F}\right)_{\mu \nu \kappa \lambda}$. These terms may assume any value, but as expected from experience with Maxwell's electrodynamics, such terms must be very small, or even, if not almost zero, immeasurable, since the symmetry violations parametrized by them have never been detected on the energy scales to which we have access nowadays. These tensors are called background fields because they spread all over spacetime, but we do not have access to their sources and we do not know their dynamics. They are fixed tensor fields that select privileged directions in spacetime, breaking its isotropy.

Terms that violate Lorentz symmetry fall into two groups. One is parametrized by terms that also violate the CPT symmetry, and another class is one preserving this symmetry. The SME gauge sector contains a term that violates the CPT symmetry, $\epsilon_{\mu \nu \alpha \beta} V^{\mu} A^{\nu} F^{\alpha \beta}$, called the odd CPT, and another term that does not violate this symmetry, $\left(K_{F}\right)_{\mu \nu \kappa \lambda} F^{\mu \nu} F^{\kappa \lambda}$, 
called the even CPT. As a result of these terms, the SME gauge sector can be divided into CPT-odd and -even and CPT-even classes.

\section{The CPT-even gauge sector of the SME with minimum length}

In this section, we shall carry out a study of the anisotropies generated by the deformation of the algebra along with the terms that violate the Lorentz symmetry. The Lagrangian density of the CPT-even gauge sector of the SME [61] is

$\mathcal{L}=-\frac{1}{4 \mu_{0}} F_{\mu \nu} F^{\mu \nu}-\frac{1}{4 \mu_{0}}\left(K_{F}\right)_{\mu \nu \kappa \lambda} F^{\mu \nu} F^{\kappa \lambda}-A_{\mu} J^{\mu}$,

where $\left(K_{F}\right)_{\mu \nu \kappa \lambda}$ is a dimensionless coupling tensor responsible for the violation of the Lorentz symmetry. It has the same symmetries as the Riemann tensor, i.e.

$$
\begin{gathered}
\left(K_{F}\right)_{\mu \nu \kappa \lambda}=-\left(K_{F}\right)_{\nu \mu \kappa \lambda}, \quad\left(K_{F}\right)_{\mu \nu \kappa \lambda} \\
=-\left(K_{F}\right)_{\mu \nu \lambda \kappa}, \quad\left(K_{F}\right)_{\mu \nu \kappa \lambda}=\left(K_{F}\right)_{\kappa \lambda \mu \nu} ; \\
\left(K_{F}\right)_{\mu \nu \kappa \lambda}+\left(K_{F}\right)_{\mu \kappa \lambda \nu}+\left(K_{F}\right)_{\mu \lambda \nu \kappa}=0
\end{gathered}
$$

and a null double trace,

$\left(K_{F}\right)_{\mu \nu}^{\mu \nu}=0$.

We write this Lagrangian in the presence of the minimum length using (5), i.e.,

$$
\begin{aligned}
& x^{\mu} \rightarrow X^{\mu}=x^{\mu}, \\
& \partial^{\mu} \rightarrow \nabla^{\mu}=\left(1+\beta \hbar^{2} \square\right) \partial^{\mu},
\end{aligned}
$$

with $\square=\partial_{\mu} \partial^{\mu}$. Neglecting terms of order higher than 1 in $\beta$, we obtain

$$
\begin{aligned}
\mathcal{L} & =-\frac{1}{4 \mu_{0}} F_{\mu \nu} F^{\mu \nu}-\frac{1}{4 \mu_{0}}\left(K_{F}\right)_{\mu \nu \kappa \lambda} F^{\mu \nu} F^{\kappa \lambda}-A_{\mu} J^{\nu}+ \\
& -\frac{1}{2 \mu_{0}} \beta \hbar^{2} F_{\mu \nu} \square F^{\mu \nu}-\frac{1}{2 \mu_{0}} \beta \hbar^{2}\left(K_{F}\right)_{\mu \nu \kappa \lambda} F^{\mu \nu} \square F^{\kappa \lambda},
\end{aligned}
$$

from which we have the field equations

$$
\left(1+2 \beta \hbar^{2} \square\right)\left[\partial_{\nu} F^{\nu \mu}-\left(K_{F}\right)_{\rho \phi}^{\mu \nu} \partial_{\nu} F^{\rho \phi}\right]=\mu_{0} J^{\mu} .
$$

Let us recall that

$$
\partial_{\nu} F_{\alpha \beta}+\partial_{\alpha} F_{\beta \nu}+\partial_{\beta} F_{\nu \alpha}=0 .
$$

If we take $v=i, \alpha=j, \beta=l$ in (14), we get the equation $\nabla \cdot \vec{B}=0$ then, for $v=0, \alpha=i, \beta=j$ in (14), we obtain the Faraday law,

$\nabla \times \vec{E}=-\frac{\partial \vec{B}}{\partial t}$.

A useful parameterization for this theory is that described in $[62,63]$, where the 19 independent components of the tensor $\left(K_{F}\right)$ are contained in four $3 \times 3$ matrices: $\left(\kappa_{D E}\right),\left(\kappa_{H B}\right)$, $\left(\kappa_{D B}\right)$ and $\left(\kappa_{H E}\right)$.

The components of these matrices are given by

$$
\begin{gathered}
\left(\kappa_{D E}\right)^{j k}=-2\left(K_{F}\right)^{0 j 0 k}, \\
\left(\kappa_{H B}\right)^{j k}=\frac{1}{2} \epsilon^{j p q} \epsilon^{k l m}\left(K_{F}\right)^{p q l m}, \\
\left(\kappa_{D B}\right)^{j k}=-\left(\kappa_{H E}\right)^{k j}=\epsilon^{k p q}\left(K_{F}\right)^{0 j p q} .
\end{gathered}
$$

From this we see that $\left(\kappa_{D E}\right)$ and $\left(\kappa_{H B}\right)$ are symmetric, while $\left(\kappa_{D B}\right)$ does not exhibit symmetry. With this in mind, taking $\mu=0$ in (13) we get the modified Gaussian law

$$
\left(1+2 \beta \hbar^{2} \square\right)\left[\partial_{i} E^{i}+\left(\kappa_{D E}\right)_{l j} \partial_{l} E^{j}+c\left(\kappa_{D B}\right)_{l k} \partial_{l} B_{k}\right]=\frac{\rho}{\epsilon_{0}},
$$

and taking $\mu=i$ in (13), we have the modified AmpèreMaxwell law

$$
\begin{aligned}
& \left(1+2 \beta \hbar^{2} \square\right)\left[-\partial_{t} E^{i} / c^{2}+\epsilon_{i j k} \partial_{j} B^{k}\right. \\
& -\left(\kappa_{D E}\right)_{i j} \partial_{t} E^{j} / c^{2}+\left(\kappa_{D B}\right)_{i k} \partial_{t} B^{k} / c+ \\
& \left.\left(\kappa_{H B}\right)_{j k} \epsilon_{j i p} \partial_{p} B^{k}+\epsilon_{i p k}\left(\kappa_{D B}\right)_{m k} \partial_{p} E^{m} / c\right]=\mu_{0} J^{i} .
\end{aligned}
$$

We see then that, in vacuum $\left(J^{\mu}=0\right)$, the minimum length modifies the Gauss and Ampère-Maxwell laws by the global phase factor $\left(1+2 \beta \hbar^{2} \square\right)$. The dispersion relation calculated from (13) yields, in addition to the usual modes obtained in the absence of minimum length, the mode

$$
1+2 \beta \hbar^{2} \square=0,
$$

i.e.

$p^{0^{2}}=\vec{p}^{2}+\frac{1}{2 \beta}$,

which describes a particle of mass $m=1 / \sqrt{2 \beta}$.

We conclude that, for the CPT-even gauge sector of the SME, the phenomena of LSV and minimum length are independent of each other, that is, even for an electrodynamics that does not spontaneously violate the Lorentz symmetry $\left(K_{F}=0\right)$ it would exhibit a massive pole as shown in [64]. 


\section{The CPT-odd gauge sector of the SME with minimum length}

The Lagrangian density of the CPT-odd gauge sector of the SME is given by

$$
\mathcal{L}=-\frac{1}{4 \mu_{0}} F_{\mu \nu} F^{\mu \nu}-\frac{\chi}{4 \mu_{0}} \epsilon_{\mu \nu \alpha \beta} V^{\mu} A^{\nu} F^{\alpha \beta}-A_{\mu} J^{\mu}
$$

where $V^{\mu}$ is a fixed background field (and $\chi$ is a constant such that $\left.\operatorname{dim}\left(\chi V^{\mu}\right)=1 /[L]\right)$ and it is responsible for the violation of the Lorentz symmetry [65].

Let us write, as in the CPT-even case, this Lagrangian in the presence of the minimum length using (5); neglecting terms of order higher than 1 in $\beta$, we obtain

$$
\begin{aligned}
\mathcal{L} & =-\frac{1}{4 \mu_{0}} F_{\mu \nu} F^{\mu \nu}-\frac{\chi}{4 \mu_{0}} \epsilon_{\mu \nu \alpha \beta} V^{\mu} A^{\nu} F^{\alpha \beta}-A_{\mu} J^{\nu}+ \\
& -\frac{1}{2 \mu_{0}} \beta \hbar^{2} F_{\mu \nu} \square F^{\mu \nu}-\frac{\chi}{4 \mu_{0}} \beta \hbar^{2} \epsilon_{\mu \nu \alpha \beta} V^{\mu} A^{\nu} \square F^{\alpha \beta},
\end{aligned}
$$

and then the equations of motion follow:

$$
\begin{aligned}
& \left(1+2 \beta \hbar^{2} \square\right) \partial_{\nu} F^{\nu \mu} \\
& \quad+\frac{\chi}{2} \epsilon^{\mu \lambda \alpha \beta} V_{\lambda}\left(1+\beta \hbar^{2} \square\right) F_{\alpha \beta}=\mu_{0} J^{\mu}, \\
& \partial_{\nu} F_{\alpha \beta}+\partial_{\alpha} F_{\beta \nu}+\partial_{\beta} F_{\nu \alpha}=0 .
\end{aligned}
$$

From (23), we obtain the modified Gauss and AmpèreMaxwell laws

$$
\begin{aligned}
& \left(1+2 \beta \hbar^{2} \square\right) \nabla \cdot \vec{E}-c \chi \vec{V} \cdot\left(1+2 \beta \hbar^{2} \square\right) \vec{B}=\frac{\rho}{\epsilon_{0}} \\
& \left(1+2 \beta \hbar^{2} \square\right)\left(\nabla \times \vec{B}-\frac{1}{c^{2}} \frac{\partial \vec{E}}{\partial t}\right)-\chi V_{0}\left(1+\beta \hbar^{2} \square\right) \vec{B} \\
& \quad+\chi \vec{V} \times\left(1+\beta \hbar^{2} \square\right) \frac{\vec{E}}{c}=\mu_{0} \vec{J},
\end{aligned}
$$

while (24) gives us the homogeneous equations of Maxwell

$\nabla \cdot \vec{B}=0$

$$
\frac{\partial \vec{B}}{\partial t}+\nabla \times \vec{E}=0
$$

We then see that, unlike what happens in the CPT-even gauge sector of the SME (in vacuum), here the equations of motion are not modified by a global phase factor. Starting from (23), using the Lorenz gauge $\partial^{\sigma} A_{\sigma}=0$, we obtain

$$
\left(1+2 \beta \hbar^{2} \square\right) \square A_{\mu}+\frac{\chi}{2}\left(1+\beta \hbar^{2} \square\right) \epsilon_{\lambda \mu \alpha \beta} V^{\lambda} F^{\alpha \beta}=\mu_{0} J_{\mu},
$$

which can be rewritten in matrix form as

$M_{\lambda \beta} A^{\beta}=\mu_{0} J_{\lambda}$

where

$M_{\lambda \beta}=\left(1+2 \beta \hbar^{2} \square\right) \square \eta_{\lambda \beta}+\chi\left(1+\beta \hbar^{2} \square\right) \epsilon_{\lambda \mu \alpha \beta} V^{\mu} \partial^{\alpha}$.

Working in Fourier space (of momenta), it follows that

$$
\begin{aligned}
A^{\beta} & =\widetilde{A}^{\beta} \exp (-i p \cdot x / \hbar) \\
& =\widetilde{A}^{\beta} \exp \left[\left(-i p_{0} t+i \vec{p} \cdot \vec{x}\right) / \hbar\right], \\
\partial_{\mu} & \rightarrow-\frac{i}{\hbar} p_{\mu}, \quad \partial_{\mu} \partial^{\mu} \rightarrow-\frac{p^{2}}{\hbar^{2}}
\end{aligned}
$$

then

$$
M_{\lambda \beta}(p)=-\left(1-2 \beta p^{2}\right) \frac{p^{2}}{\hbar^{2}} \eta_{\lambda \beta}-i \frac{\chi}{\hbar}\left(1-\beta p^{2}\right) \epsilon_{\lambda \mu \alpha \beta} V^{\mu} p^{\alpha}
$$

By means of extensive algebraic manipulations, we obtain the determinant of the matrix $M$, that is,

$$
\begin{aligned}
\operatorname{det}(M)= & \left(1-2 \beta p^{2}\right)^{2} \frac{p^{4}}{\hbar^{4}}\left\{-\left(1-2 \beta p^{2}\right)^{2} \frac{p^{4}}{\hbar^{4}}\right. \\
& \left.-\left(1-\beta p^{2}\right)^{2} \frac{\chi^{2}}{\hbar^{2}}\left[V^{2} p^{2}-(V \cdot p)^{2}\right]\right\} .
\end{aligned}
$$

The dispersion relation is obtained by taking $\operatorname{det}(M)=0$; then two cases show up.

\section{Case 1:}

$\left(1-2 \beta p^{2}\right)^{2} \frac{p^{4}}{\hbar^{4}}=0$.

In this case, we obtain the mode $p=0$, which is the ordinary photon, and $p^{2}=1 / 2 \beta$, that is,

$p^{0^{2}}=\vec{p}^{2}+\frac{1}{2 \beta}$,

which is the same as Eq. (20), with a particle of mass $m=$ $1 / \sqrt{ }(2 \beta) c$.

\section{Case 2:}

$$
\left(1-2 \beta p^{2}\right)^{2} \frac{p^{4}}{\hbar^{4}}+\left(1-\beta p^{2}\right)^{2} \frac{\chi^{2}}{\hbar^{2}}\left[V^{2} p^{2}-(V \cdot p)^{2}\right]=0
$$

as we are only considering terms of up to order 1 in $\beta$ we get 


$$
\begin{aligned}
& 4 \beta p^{6}+\left(2 \beta \bar{\chi} V^{2}-1\right) p^{4} \\
& -\bar{\chi}\left[V^{2}+2 \beta(V \cdot p)^{2}\right] p^{2}+\bar{\chi}^{2}(V \cdot p)^{2}=0,
\end{aligned}
$$

where $\bar{\chi}=\chi \hbar$.

Equation (39) contains terms that are products between the deformation parameter $\beta$ and the $\mathrm{LV}$ vector $\vec{V}$. Therefore, it would provide a mode of propagation in which the minimum length and LSV would occur simultaneously, being dependent on each other, that is, we would have an expression that relates these two phenomena. It indicates a possible justification of why both the minimum length and the violation of Lorentz symmetry have never been detected experimentally (all we have are upper bounds on the parameters), since a mode of propagation that presents a term of the form " $\beta V$ ", the product of two very small magnitudes, is also very tiny.

Unfortunately, Eq. (39) is of degree 6 and does not present a solution of the form $p=p(\beta, V)$ (in analogy to the case with no minimum length where the solution is of the form $p=p(V)$ ). Therefore, as in the CPT-even gauge sector of the SME, we conclude that the phenomena of LSV and minimum length are independent of each other.

\section{Concluding remarks}

We have studied the gauge sector of SME by taking into account the presence of a minimum length. We have analyzed the dispersion relations of this theory. Our motivation for such a study comes from the fact that both, the violation of Lorentz symmetry and the presence of a minimum length, have never, as previously stated, detected experimentally. All we have are upper limits, and the hypothesis that these two phenomena could occur simultaneously and dependent on each other, which is revealed through a dispersion relation where the terms violating Lorentz symmetry appear multiplied by the minimum length deformation parameter. It would justify this difficulty of detection, since the quantity to be measured would be the product of two very small quantities and, therefore, much smaller. However, the dispersion relations showed us that there are no modes of propagation that simultaneously contain contributions from the $\mathrm{LV}$ and minimum length. We have obtained, in addition to the usual propagation modes obtained in the absence of minimum length, the mode described by Eq. (20), which describes a particle of mass $m=1 / \sqrt{2 \beta} c$, widely discussed in [64]. Based on studies of the anomalous magnetic moment of the electron [66-68], the estimates that have been found were $\left(\Delta X^{i}\right)_{0} \leq 7.4 \times 10^{-18} \mathrm{~m}$, close to the electroweak scale $l_{\text {electroweak }} \approx 10^{-18} \mathrm{~m}$ ) and $l_{\text {electroweak }} \approx 10^{-18} \mathrm{~m}$ (same order of magnitude of the masses of the vector bosons $W^{ \pm}$and $Z^{0}$ ), and, on the basis of studies of the fundamental state of the hydrogen atom [69], they obtained $\left(\Delta X^{i}\right)_{0} \leq 8.79 \times 10^{-15} \mathrm{~m}$ (close to the scale of strong interactions $l_{\text {strong }} \approx 10^{-15} \mathrm{~m}$ ) and $m \geq 35.51 \frac{\mathrm{MeV}}{c^{2}}$.

It is important to remember that, in our investigation, we have supposed the violation of the Lorentz symmetry to occur spontaneously, i.e., due only to the background tensors. We have introduced the minimum length by means of a Lorentz covariant deformed algebra, with these two initial assumptions. Then we have seen that these phenomena can be taken as independent. Another approach would be to introduce the minimum length by means of a deformed algebra that violates the Lorentz symmetry (i.e., a non-covariant algebra). Thus, the two phenomena would already be connected from the very beginning. The study of how the violation of Lorentz symmetry and minimum length are related to each other is a very interesting and promising subject for investigation, since the two phenomena are expected to be valid at high energies (above the Standard Model). The remaining question is whether or not there is a range of energy in which they interfere significantly.

Acknowledgements The authors thank CNPq and FAPES/ES (of Brazil) for financial support.

Open Access This article is distributed under the terms of the Creative Commons Attribution 4.0 International License (http://creativecomm ons.org/licenses/by/4.0/), which permits unrestricted use, distribution, and reproduction in any medium, provided you give appropriate credit to the original author(s) and the source, provide a link to the Creative Commons license, and indicate if changes were made. Funded by $\mathrm{SCOAP}^{3}$.

\section{References}

1. V.A. Kostelecký, S. Samuel, Phys. Rev. D 39, 683 (1989)

2. D. Colladay, V.A. Kostelecký, Phys. Rev. D 55, 6760 (1997)

3. D. Colladay, V.A. Kostelecký, Phys. Rev. D 58, 116002 (1998)

4. H. Belich, T. Costa-Soares, M.A. Santos, M.T.D. Orlando, Rev. Bras. Ens. Fís. 29, 1 (2007)

5. H. Belich, T. Costa-Soares, M.M. Ferreira Jr., J.A. Helayël-Neto, F.M.O. Moucherek, Phys. Rev. D 74, 065009 (2006)

6. G. Gazzola, H.G. Fargnoli, A.P. Baêta Scarpelli, M. Sampaio, M.C. Nemes, J. Phys. G: Nucl. Part. Phys. 39, 035002 (2012)

7. H. Belich, L.P. Collato, T. Costa-Soares, J.A. Helayël-Neto, M.T.D. Orlando, Eur. Phys. J. C 62, 425 (2009)

8. R. Casana, M.M. Ferreira Jr., V.E. Mouchrek-Santos, E.O. Silva, Phys. Lett. B 746, 171 (2015)

9. R. Casana, C.F. Farias, M.M. Ferreira, Phys. Rev. D 92, 125024 (2015)

10. R. Casana, M.M. Ferreira Jr., F.E.P. dos Santos, Phys. Rev. D 90, 105025 (2014)

11. R. Casana, M.M. Ferreira Jr., E. da Hora, A.B.F. Neves, Eur. Phys. J. C 74, 3064 (2014)

12. R. Casana, M.M. Ferreira Jr., R.V. Maluf, F.E.P. dos Santos, Phys. Lett. B 726, 815 (2013)

13. J.B. Araujo, R. Casana, M.M. Ferreira Jr., Phys. Lett. B 760, 302 308 (2016)

14. H. Belich, T. Costa-Soares, M.M. Ferreira Jr., J.A. Helayël-Neto, Eur. Phys. J. C 41, 421 (2005)

15. M.A. Ajaib, Int. J. Mod. Phys. A 27, 1250139 (2012) 
16. A.G. Grushin, Phys. Rev. D 86, 045001 (2012)

17. H. Belich, F.J.L. Leal, H.L.C. Louzada, M.T.D. Orlando, Phys. Rev. D 86, 125037 (2012)

18. R. Casana, M.M. Ferreira Jr., E. Passos, F.E.P. dos Santos, E.O. Silva, Phys. Rev. D 87, 047701 (2013)

19. H. Belich, L.P. Colatto, T. Costa-Soares, J.A. Helayël-Neto, M.T.D. Orlando, Eur. Phys. J. C 62, 425 (2009)

20. H. Belich, E.O. Silva, M.M. Ferreira Jr., M.T.D. Orlando, Phys. Rev. D 83, 125025 (2011)

21. L.R. Ribeiro, E. Passos, C. Furtado, J.R. Nascimento, Int. J. Mod. Phys. A 30, 1550072 (2015)

22. K. Bakke, H. Belich, J. Phys. G Nucl. Part. Phys. 40, 065002 (2013)

23. K. Bakke, H. Belich, J. Phys. G Nucl. Part. Phys. 39, 085001 (2012)

24. K. Bakke, H. Belich, Spontaneous Lorentz Symmetry Violation and Low Energy Scenarios (LAMBERT Academic Publishing, Saarbrücken, 2015)

25. L.R. Ribeiro, E. Passos, C. Furtado, J. Phys. G Nucl. Part. Phys. 39, 105004 (2012)

26. Q.G. Bailey, V.A. Kostelecký, Phys. Rev. D 74, 045001 (2006)

27. V.A. Kostelecký, J.D. Tasson, Phys. Rev. Lett. 102, 010402 (2009)

28. V.A. Kostelecký, Phys. Rev. D 69, 105009 (2004)

29. H.S. Snyder, Phys. Rev. 71, 38 (1947)

30. C.N. Yang, Phys. Rev. 72, 874 (1947)

31. A. Connes, $C^{*}$ algebras and differential geometry. Compt. Rend Acad. Sci. (Ser. I Math.) A290, 599-609 (1980)

32. A. Connes, Michael R. Douglas, Albert Schwarz, JHEP 02, 003 (1998)

33. Michael R. Douglas, Nikita A. Nekrasov, Rev. Mod. Phys. 73, $977-$ 1029 (2001)

34. Michael R. Douglas, Christopher M. Hull, JHEP 02, 008 (1998)

35. G. Amelino-Camelia, Nature 418, 34-35 (2002). arXiv:gr-qc/0207049

36. G. Amelino-Camelia, Int. J. Mod. Phys. D 11, 1643 (2002). arXiv:gr-qc/0210063

37. Y. Xu, B.-Q. Ma, Mod. Phys. Lett. A 26, 2101-2108 (2011). arXiv: 1106.1778

38. B.-Q. Ma, Mod. Phys. Lett. A 28, 1340012 (2013). arXiv: 1203.5852

39. A. Kempf, J. Math. Phys. 35, 4483 (1994)

40. A. Kempf, G. Mangano, R.B. Mann, Phys. Rev. D 52, 1108 (1995)

41. H. Hinrichsen, A. Kemph, J. Math. Phys. 37, 2121 (1996)

42. A. Kempf, J. Math. Phys. 38, 1347 (1997)

43. A. Kempf, J. Phys. A 30, 2093 (1997)
44. D.J. Gross, P.F. Mende, Nucl. Phys. B 303, 407 (1988)

45. M. Maggiore, Phys. Lett. B 304, 65 (1993)

46. E. Witten, Phys. Today 49, 24 (1996)

47. S.G. Karshenboim, Phys. Rep. 422, 1 (2005)

48. F. Brau, J. Phys. A 32, 7691 (1999)

49. S. Benczik, L.N. Chang, D. Minic, T. Takeuchi, Phys. Rev. A 72, 012104 (2005)

50. R. Akhoury, Y.-P. Yao, Phys. Lett. B 572, 37 (2003)

51. C. Quesne, V.M. Tkachuk, J. Phys. A Math. Gen. 39, 10909 (2006)

52. C. Quesne, V.M. Tkachuk, Czech. J. Phys. 56, 1269 (2006)

53. V.M. Tkachuk, J. Phys. Stud. 11, 41 (2007)

54. G. Betschart, E. Kant, F.R. Klinkhamer, Nucl. Phys. B 815, 198 $214(2009)$

55. E. Kant, F.R. Klinkhamer, M. Schreck, Phys. Lett. B 682, 316-321 (2009)

56. D. Colladay, V.A. Kostelecký, Phys. Rev. D 55, 6760 (1997)

57. D. Colladay, V.A. Kostelecký, Phys. Rev. D 58, 116002 (1998)

58. H. Belich, T. Costa-Soares, M.M. Ferreira Jr., J.A. Helayël-Neto, Eur. Phys. J. C 41, 421 (2005)

59. H. Belich, L.P. Collato, T. Costa-Soares, J.A. Helayël-Neto, M.T.D. Orlando, Eur. Phys. J. C 62, 425 (2009)

60. K. Bakke, H. Belich, E.O. Silva, Ann. Phys. (Berl.) 523, 910 (2011)

61. R. Casana, M.M. Ferreira Jr., R.P.M. Moreira, Phys. Rev. D 84, 125014 (2011)

62. V.A. Kostelecký, M. Mewes, Phys. Rev. Lett. 87, 251304 (2001)

63. V.A. Kostelecky, Matthew Mewes, Phys. Rev. D 66, 056005 (2002)

64. S.K. Moayedi, M.R. Setare, B. Khosropour, Adv. High Energy Phys. 2013, 657870 (2013)

65. S.M. Carroll, G.B. Field, R. Jackiw, Phys. Rev. D 41(4), 1231 (1990)

66. A. Accioly, E. Scatena, Mod. Phys. Lett. A 25, 269 (2010)

67. A. Accioly, P. Gaete, J. Helayel-Neto, E. Scatena, R. Turcati, Mod. Phys. Lett. A 26, 1985 (2011)

68. A. Accioly, H. Mukai, Nuovo Cimento B 112, 1061 (1997)

69. R.R. Cuzinatto, C.A.M. de Melo, L.G. Medeiros, P.J. Pompeia, Int. J. Mod. Phys. A 26, 3641 (2011) 